\title{
Pseudostate description of diatomic-molecule scattering from a hard-wall potential
}

\author{
A. V. Lugovskoy* and I. Bray \\ ARC Centre for Antimatter-Matter Studies, Curtin University, Perth 6150, Australia
}

(Received 4 October 2012; published 31 January 2013)

\begin{abstract}
A collinear scattering of a structured particle from a hard wall is studied with consideration of vibrational transitions initiated by the collision. It is shown that this problem can be solved analytically in the framework of the source-function method. With the use of the continuum discretization technique we are able to take into account both discrete and continuum states. No approximations of the interatomic potential is required. We illustrate our approach for the case of a hydrogen molecule bound by the realistic Morse potential.
\end{abstract}

DOI: 10.1103/PhysRevA.87.012904

PACS number(s): 79.20.Rf, 34.50.Fa, 34.70.+e

\section{INTRODUCTION}

Controlling motion of individual atoms and molecules is of importance for pure science and technology. This level of control is required for realization of the most ambitious modern-day technological projects such as quantum information processing and quantum simulation [1]. One way of mastering the quantum world is to utilize the dependence of the transport properties of atoms and molecules on their inner degrees of freedom [2]. Superconductivity and laser cooling of atoms are classical examples that demonstrate how vital the intrinsic degrees of freedom can be.

Diatomic-molecule-like composite particles have recently attracted attention due to the peculiar intrinsic structure dependence of their transport properties resulting in anomalous diffusion in random potential fields [3-5], resonant tunneling of molecules through potential barriers [6-9], and resonant tunneling of molecules in one-dimensional lattices [10]. Being of a general nature, such phenomena do not belong solely to the domain of molecular physics. The same principles work in other branches of quantum science. Indeed, phenomena similar to resonant tunneling [6] can be seen at the internuclear level $[11,12]$.

Thus far, the approaches used in the description of the above scattering processes have been very simplistic. They are limited to generally the one-dimensional case with the use of unrealistic interaction potentials that allowed for analytical evaluation. In these problems the molecular energies are small, which should require a quantum-mechanical treatment of both the internuclear and molecular motion. The analysis relies upon the direct solution of the Schrödinger equation with the chosen simplified potentials. Generally, the molecular continuum (breakup) is not taken into account in such calculations.

In this paper we will deal with scattering of a composite particle (molecule) from a hard-wall potential. Without loss of generality we will consider the one-dimensional case where the direction of the molecule translational motion is collinear with the diatomic molecular axis. Also, we assume that the molecule velocity is normal to the surface. Previously, a similar problem was studied numerically by Sato and Kayanuma [13] for the case of the harmonic binding potential and analytically by Kavka et al. [9] for a molecule bound by a $\delta$-function potential. These studies revealed some unexpected behavior for such

\footnotetext{
*a.lugovskoy@curtin.edu.au
}

simple systems. The results by Sato and Kayanuma [13] indicate that the initial molecule ground state cannot survive after the collision when the energy difference between this state and the first-excited state goes to zero. Contrarily, Kavka et al. [9] show that an arbitrarily weakly bound molecule scattering from an arbitrarily high step potential remains in the ground state with probability equal to unity. Moreover, the molecule center of mass cannot get closer to the hard wall than $\xi_{0} \ln \left(V_{0} / E_{\text {in }}\right)$ where $\xi_{0}$ is a constant of order of the mean distance between the atoms, $V_{0}$ is the potential barrier heights and $E_{\text {in }}$ is the molecule impact energy. The case where the hard wall is infinitely high is particularly interesting because the molecule reflects from the surface at an infinitely large distance from the surface.

In the next section we present the general analytical solution to the problem of interest for case where $V_{0}$ is infinite. Our approach requires no approximations on the form of the interparticle interaction. To illustrate our theory we give a numerical example in Sec. III. In the conclusion we present the summary of the results.

\section{THEORETICAL MODEL}

In this section we study theoretically how a diatomic molecule scatters from a hard-wall barrier. The molecule is treated as two atomic particles, each one of them having mass $m$, bound together with some potential $V_{a}$. We limit our study to the case where the interatomic motion and motion of the molecule as a whole are aligned and perpendicular to the surface, reducing the problem to just one spatial dimension. Such assumptions are readily relaxed. The position of the molecule as a whole is specified with $x=\left(x_{1}+x_{2}\right) / 2$ where $x_{i}$ is the coordinate of the $i$ th atom with respect to the hard wall. The interatomic separation is $\xi=x_{1}-x_{2}$. We assume that the molecule is initially in the vibrational state with quantum number $v_{\text {in }}$ with energy $\epsilon_{v_{\text {in }}}$. The total energy of the system is $E=E_{\text {in }}+\epsilon_{v_{\text {in }}}$, where $E_{\text {in }}=k_{\text {in }}^{2} /(2 M), k_{\text {in }}$ and $M=2 m$ are the impact energy, the initial wave number, and the mass of the molecule, respectively. Atomic units are used throughout unless specified otherwise.

Let the molecule propagate from $x=-\infty$ to the hard-wall barrier $V_{\mathrm{hw}}$ at $x=0$. The Hamiltonian of the system is

$$
\begin{aligned}
H= & -\frac{1}{2 m} \frac{\partial^{2}}{\partial x_{1}^{2}}-\frac{1}{2 m} \frac{\partial^{2}}{\partial x_{2}^{2}}+V_{a}\left(\left|x_{1}-x_{2}\right|\right) \\
& +V_{\mathrm{hw}}\left(x_{1}\right)+V_{\mathrm{hw}}\left(x_{2}\right) .
\end{aligned}
$$


Alternatively, it can be written as

$$
\begin{aligned}
H= & -\frac{1}{2 M} \frac{\partial^{2}}{\partial x^{2}}-\frac{1}{2 \mu} \frac{\partial^{2}}{\partial \xi^{2}}+V_{a}(|\xi|) \\
& +V_{\mathrm{hw}}\left(x+\frac{\xi}{2}\right)+V_{\mathrm{hw}}\left(x-\frac{\xi}{2}\right),
\end{aligned}
$$

where $\mu=m / 2$ is the reduced mass of the molecule. The initial state wave function

$$
\Psi_{\text {in }}(x, \xi)=\phi_{\nu_{\text {in }}}(\xi) \exp \left[i k_{\text {in }} x\right]
$$

is an eigenfunction of the unperturbed Hamiltonian

$$
H_{0}=-\frac{1}{2 M} \frac{\partial^{2}}{\partial x^{2}}-\frac{1}{2 \mu} \frac{\partial^{2}}{\partial \xi^{2}}+V_{a}(|\xi|) .
$$

In Eq. (3), the function $\phi_{v}$ describes the vibrational state of the molecule. The plane-wave term describes the molecule propagation as a whole.

Hamiltonian $H$ is invariant under mutual permutations of $x_{1}$ and $x_{2}$. This results in the fact that the solution $\psi$ of the Schrödinger equation

$$
(H-E) \psi\left(x_{1}, x_{2}\right)=0
$$

must be symmetric $\left[\psi\left(x_{1}, x_{2}\right)=\psi\left(x_{2}, x_{1}\right)\right]$ if $\phi_{\nu_{\text {in }}}(\xi)$ is an even function of $\xi$ or asymmetric $\left[\psi\left(x_{1}, x_{2}\right)=-\psi\left(x_{2}, x_{1}\right)\right]$ if $\phi_{\nu_{\text {in }}}(\xi)$ is odd.

To find the system wave function $\psi$ satisfying the boundary condition (3) we transform Eq. (5) to the integral form

$$
\begin{aligned}
& \psi^{( \pm)}(x, \xi) \\
& =\phi_{\nu_{\text {in }}}^{( \pm)}(\xi) e^{i k_{\text {in }} x}+\sum_{\nu} \int_{-\infty}^{\infty} d k \frac{F_{\nu}(k) \phi_{\nu}(\xi) e^{i k x}}{E^{+}-\epsilon_{\nu}-k^{2} /(2 M)},
\end{aligned}
$$

where

$$
\begin{aligned}
F_{v}(k)= & \int_{-\infty}^{\infty} d x_{1} \int_{-\infty}^{\infty} d x_{1} \phi_{v}^{*}\left(x_{1}-x_{2}\right) e^{-i k\left(x_{1}+x_{2}\right) / 2} \\
& \times\left[V_{\mathrm{hw}}\left(x_{1}\right)+V_{\mathrm{hw}}\left(x_{2}\right)\right] \psi\left(x_{1}, x_{2}\right) \\
= & \int_{-\infty}^{\infty} d x_{1} \int_{-\infty}^{\infty} d x_{1}\left[\phi_{v}^{*}\left(x_{1}-x_{2}\right) \pm \phi_{v}^{*}\left(x_{2}-x_{1}\right)\right] \\
& \times e^{-i k\left(x_{1}+x_{2}\right) / 2} V_{\mathrm{hw}}\left(x_{1}\right) \psi^{( \pm)}\left(x_{1}, x_{2}\right)
\end{aligned}
$$

and where the subscript $( \pm)$ indicates the wave function symmetry. The sum over $v$ in Eq. (6) includes both discrete and continuum states. The factor $\left[\phi_{v}^{*}(\xi) \pm \phi_{v}^{*}(-\xi)\right]$ in the integrand of the matrix element (8) ensures that only the states with the symmetry of the initial state survive in the wave function expansion (6).

In order to solve Eq. (6) we employ the source-function method developed initially by Masel et al. [14] for the problem of structureless-particle scattering from a corrugated hard-wall potential. It was generalized for the case of a structured atomic particle scattering from the hard-wall potential by Lugovskoy and Bray [15]. Following this approach we assume that the product $V_{\mathrm{hw}}\left(x_{1}\right) \psi^{( \pm)}\left(x_{1}, x_{2}\right)$ is nonzero only when the particle with the coordinate $x_{1}$ is at the barrier $\left(x_{1}=0\right)$. At this point the potential changes from zero to infinity. Thus we set

$$
V_{\mathrm{hw}}\left(x_{1}\right) \psi^{( \pm)}\left(x_{1}, x_{2}\right)=f\left(x_{2}\right) \delta\left(x_{1}\right)
$$

where $f(x)$ is the source function to be found. In addition we assume that

$$
f\left(x_{2}\right)=0 \text { when } x_{2}>0,
$$

since we assume that when one atom stops, both stop. With the use of Eqs. (9) and (10) the double integral of Eq. (8) can be reduced to the one-dimensional integral

$$
F_{\nu}(k)=\int_{-\infty}^{0} d \xi\left[\phi_{\nu}^{*}(-\xi) \pm \phi_{\nu}^{*}(\xi)\right] e^{-i k \xi / 2} f(\xi) .
$$

To calculate $f(\xi)$ we will use the fact that the molecule cannot penetrate the hard-wall-barrier. This means that $\psi(x, \xi)=$ 0 when $x>0$. Using this condition one can calculate the integral over $k$ in Eq. (6) analytically. After some algebra we get

$$
\begin{aligned}
\psi^{( \pm)}(x>0, \xi)= & \sum_{\nu} \phi_{\nu}(\xi) \exp \left[i k_{\nu} x\right] \\
& \times\left(\delta_{\nu, \nu_{\text {in }}}-\frac{2 M \pi i}{k_{v}} F_{\nu}\left(k_{v}\right)\right)=0,
\end{aligned}
$$

where $k_{v}=\sqrt{2 M\left(E-\epsilon_{v}\right)}$. Note that summation in Eq. (12) [and (13)] is carried out over both open $\left(E>\epsilon_{v}\right)$ and closed $\left(E<\epsilon_{\nu}\right)$ states including continuum states. By multiplying Eq. (12) by $\phi_{v}^{*}(\xi)$ and integrating the product over $\xi$ one obtains

$$
\exp \left[i k_{\nu} x\right]\left(\delta_{\nu, v_{\text {in }}}-\frac{2 M \pi i}{k_{\nu}} F_{\nu}\left(k_{v}\right)\right)=0 .
$$

For Eq. (12) to be satisfied for any $x>0$, we must have

$$
F_{v}\left(k_{v}\right)=-\frac{i}{2 \pi} \frac{k_{v}}{M} \delta_{\nu, v_{\text {in }}},
$$

which is a set of integral equations for $f(\xi)$ [see Eq. (11)]. Equation (14) shows that $F_{v}\left(k_{v}\right)$ is a pure imaginary number for $v=v_{\text {in }}$, otherwise it is zero. Different channels are coupled with each other through the source function $f(\xi)$. By using Eq. (14) one can show that $f(\xi)$ takes generally complex values. These equations can be solved by expanding $f(\xi)$ over some basis set [15]. In Sec. III we will consider a numerical example.

As soon as $f(\xi)$ is calculated one is able to reconstruct $\psi^{( \pm)}(x, \xi)$ for $x<0$ with the use of Eqs. (6) and (11). Explicitly, it is

$$
\begin{aligned}
\psi^{( \pm)}(x, \xi)= & \phi_{\nu_{\text {in }}}^{( \pm)}(\xi) \exp \left[i k_{\text {in }} x\right]-2 M \pi i \underbrace{}_{\nu} \phi_{\nu}(\xi) \\
& \times\left[\frac{e^{i k_{v} x}}{k_{v}} \int_{-\infty}^{2 x} d \xi\left[\phi_{\nu}^{*}(-\xi) \pm \phi_{v}^{*}(\xi)\right] e^{-i k_{\nu} \xi / 2} f(\xi)\right. \\
& \left.+\frac{e^{-i k_{v} x}}{k_{v}} \int_{2 x}^{0} d \xi\left[\phi_{v}^{*}(-\xi) \pm \phi_{v}^{*}(\xi)\right] e^{i k_{\nu} \xi / 2} f(\xi)\right]
\end{aligned}
$$

If $x$ tends to $-\infty$ the first integral in the square brackets of Eq. (15) disappears and

$$
\begin{aligned}
\psi^{( \pm)}(x, \xi) \rightarrow & \phi_{\nu_{\text {in }}}^{( \pm)}(\xi) \exp \left[i k_{\text {in }} x\right] \\
& -\sum_{\nu: \operatorname{Im}\left(k_{v}\right)=0} C_{\nu}\left(k_{\text {in }}\right) \phi_{\nu}(\xi) \exp \left[-i k_{\nu} x\right]
\end{aligned}
$$


where $C_{v}\left(k_{\text {in }}\right)=2 M \pi i F_{v}\left(-k_{v}\right) / k_{v}$ is a function of $k_{\text {in }}$ (or $\left.E_{\text {in }}\right)$. Summation in Eq. (16) is carried out over the open states only. Finally, with the use of the asymptotic form (16) one can define the partial cross sections for the considered onedimensional case as follows:

$$
\sigma_{\nu}\left(E_{\text {in }}\right) \equiv\left|C_{\nu}\left(k_{\text {in }}\right)\right|^{2} k_{\nu} / k_{\text {in }}=4 M^{2} \pi^{2} \frac{\left|F_{\nu}\left(-k_{v}\right)\right|^{2}}{k_{\nu} k_{\text {in }}} .
$$

The conservation of the flux before and after the collision results in the relation [15]

$$
\sum_{v: \operatorname{Im}\left(k_{v}\right)=0} \sigma_{\nu}\left(E_{\text {in }}\right)=1
$$

Note that the cross sections (17) are dimensionless in our case.

\section{NUMERICAL EXAMPLE}

In this section we apply the theory developed in Sec. II to describe normal collinear scattering of a hydrogen molecule from a chemically inert solid surface, e.g., LiF. At low impact energies the molecule-surface interaction can be well approximated with the hard-wall potential [16]. The interatomic potential is taken to be the Morse potential [17]

$$
V_{a}(|\xi|)=D_{e}\left(e^{-2 a\left(|\xi|-\xi_{0}\right)}-2 e^{-a\left(|\xi|-\xi_{0}\right)}\right),
$$

where $D_{e}=0.1745$ a.u., $a=0.979$ a.u., and $\xi_{0}=1.4$ a.u. In the one-dimensional case this potential is symmetric with respect to the transformation $\xi \rightarrow-\xi$ where $\xi$ can take values from $-\infty$ to $\infty$. It results in the fact that the eigenfunctions are either even or odd.

The Morse potential (19) supports both discrete and continuum states. In this work we take all of them into account on an equal footing in the framework of the continuum discretization technique. This method was adopted from the theory of electron-atom collisions [18]. Within this method the intermolecular state is described with the finite set $\left\{\phi_{v}^{N}\right\}$ of pseudostates $\phi_{v}^{N}$ where the number of pseudostates, $N$, is a variable parameter.

The pseudostates $\phi_{v}^{N}$ and corresponding pseudoenergies $\epsilon_{v}^{N}$ are obtained upon diagonalization of the Hamiltonian

$$
H_{m}=-\frac{1}{2 \mu} \frac{d^{2}}{d \xi^{2}}+V_{a}(|\xi|)
$$

in some truncated orthogonal basis $\left\{\chi_{n}\right\}_{N}$ of size $N$. That is, they satisfy

$$
\left\langle\phi_{v}^{N}\left|H_{m}\right| \phi_{v^{\prime}}^{N}\right\rangle=\epsilon_{v}^{N} \delta_{v, v^{\prime}}
$$

where

$$
\phi_{\nu}^{N}=\sum_{\kappa=1}^{N} C_{v, \kappa} \chi_{\kappa}(\xi)
$$

with $\chi_{\kappa}(\xi)$ being a basis function and $C_{v, \kappa}$ being unknown coefficients to be calculated from Eq. (21). In this work we use the Hermite basis set with

$$
\chi_{\kappa}(\xi)=\sqrt{\frac{\lambda}{\pi^{1 / 2} 2^{\kappa} \kappa !}} H_{\kappa}(\lambda \xi) \exp \left[-\frac{(\lambda \xi)}{2}\right],
$$

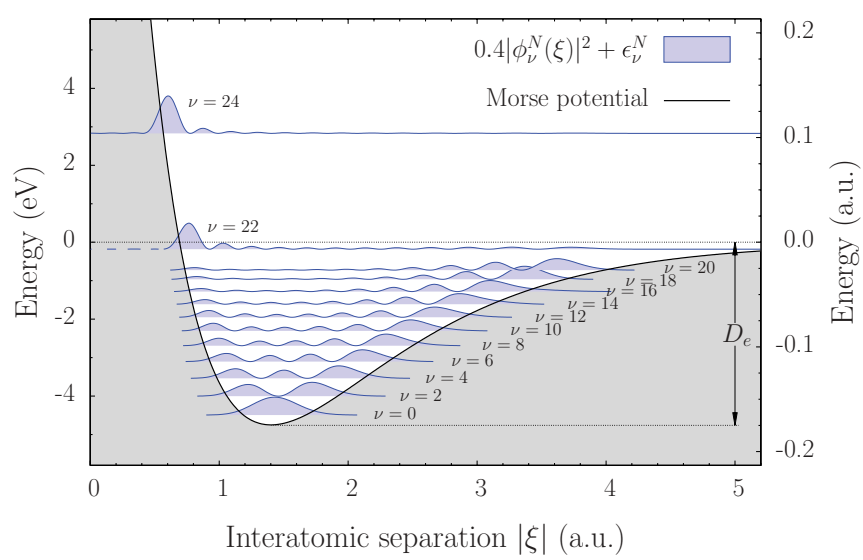

FIG. 1. (Color online) The plot of the interatomic potential together with the densities of the pseudostates calculated with $N=30$ and $\lambda=2$. Each pseudostate density $\left|\phi_{v}^{N}\right|^{2}$, shown with the filled curves, is positioned at the corresponding pseudoenergy $\epsilon_{v}^{N}$. The dark (gray) area is the classically inaccessible region.

where $H_{\kappa}(\lambda \xi)$ is the orthogonal Hermite polynomial of degree $\kappa$ and $\lambda$ is an arbitrary parameter.

Pseudostates mimic true eigenstates in the vicinity of the equilibrium bond distance, $\xi_{0}$ [19]. What is important is that the set of generated pseudo wave functions forms a finite orthonormal basis $\left\{\phi_{v}^{N}\right\}$. With increasing $N$, negative-energy pseudostates converge to true negative-energy eigenstates of the Hamiltonian (20). The parameter $\lambda$ from Eq. (23) determines the rate of convergence. The spectrum of positive pseudoenergies does not show any signs of convergence. It gets more dense and spans a wider energy region for larger values of $N$. The size of the region depends on the parameter $\lambda$. Also, it increases with $N$ [19]. All physical processes which we are able to describe with the pseudostate approach have to occur in this region.

In this work we assume that the molecule is initially in the ground vibrational state with the quantum number $v=0$. Figure 1 shows the pseudostate density $\left|\phi_{\nu=0}^{N}\right|^{2}$ together with the densities of the other states which can be found in the wave function expansion (6). They were calculated by solving numerically Eq. (21) with $N=30$ and $\lambda=3$. Comparison of the found pseudoenergies with the vibrational energies of molecular hydrogen calculated by others [20,21] shows that the relative error is less than $0.5 \%$ for the two low-lying states.

One can expect that the source function $f(\xi)$ is localized in the vicinity of $\xi=-\xi_{0}$, where $\xi_{0}$ is the equilibrium distance between the atoms in the molecule. To compute $f(\xi)$ we use the set of pseudo wave functions $\left\{\phi_{v}^{N_{\text {sf }}}\right\}$ calculated with the use of the Hamiltonian diagonalization technique as described above. The basis parameters $\lambda_{\text {sf }}$ and $N_{\text {sf }}$ were different from those used in the molecular structure calculations. This gave us flexibility to ensure the fastest convergence of the results. So we write $f(\xi)$ as

$$
f(\xi)=\sum_{\nu=0}^{N / 2} a_{\nu} \phi_{\nu}^{N_{\mathrm{sf}}}(\xi)
$$

substitute this expansion into Eq. (11), and solve Eq. (14) numerically with respect to the unknown coefficients $a_{v}$ for 

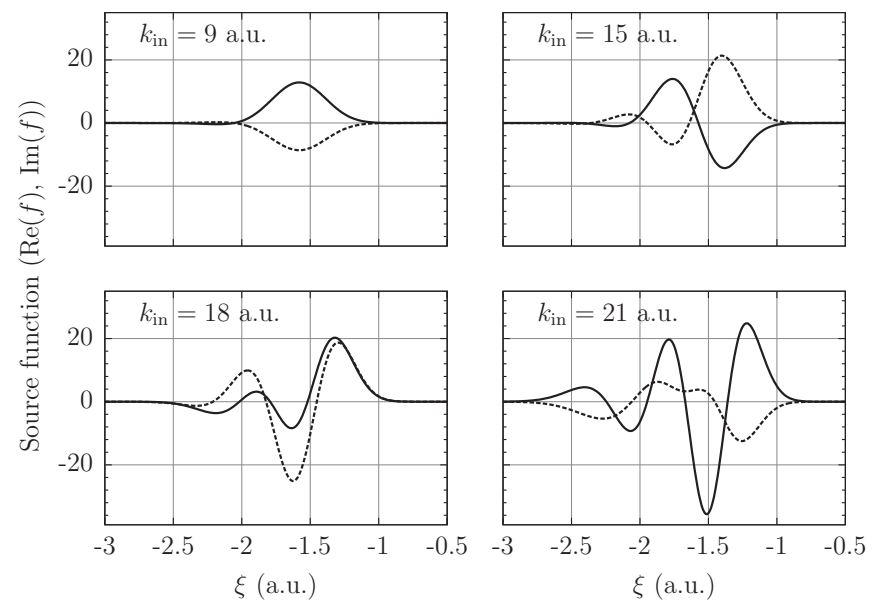

FIG. 2. Source function $f(\xi)$ calculated for four different values of the initial wave number $k_{\text {in }}$ as indicated in the figure legends. The solid (broken) line corresponds to the real (imaginary) part of the function, $\operatorname{Re}(f)$ and $\operatorname{Im}(f)$. The basis parameters were $N=60$ and $\lambda=2$ for the molecular structure and $N_{\mathrm{sf}}=30$ and $\lambda_{\mathrm{sf}}=3$ for the source function.

each impact energy $E_{\text {in }}$. Note that both even and odd solutions of Eq. (21) must be used in Eq. (24) due to the fact that the expansion basis has to be complete.

Figure 2 shows several solutions of Eq. (14) obtained with the use of the basis with $N=60$. The panel with $k_{\text {in }}=9$ corresponds to the case where only elastic scattering is possible. The other three panels illustrate the cases where only one $\left(k_{\text {in }}=\right.$ $15)$, two $\left(k_{\text {in }}=18\right)$, or three $\left(k_{\text {in }}=21\right)$ vibrational states can be excited due to the collision. The parameters of the used basis sets are given in the caption. As expected the calculated source functions $f(\xi)$ are centered around $\xi \approx-\xi_{0}=-1.4$. We see that $f(\xi)$ becomes more oscillatory for larger $k_{\text {in }}$. Also, the region where $f(\xi)$ is significantly different from zero increases with $k_{\text {in. }}$. In calculations with a limited number of expansion functions this leads to the fact that numerical solution of Eq. (14) is inaccurate when $k_{\text {in }}$ (or $E_{\text {in }}$ ) is too large.

To illustrate this we present, in Fig. 3, the elastic cross section $\sigma_{v=0}^{N}$ of a hydrogen molecule scattered from the hard-wall potential calculated with the use of three different basis sets of sizes $N=40,50$, and 60 , with $\lambda=2$. The basis parameters used to compute the source function are given in the caption. We see that all calculated cross sections are on top of each other when the incident energy $E_{\text {in }}$ is less than $\sim 1.4 \mathrm{eV}$. For larger energies $\sigma_{v=0}^{N=40}$ deviates from the cross sections calculated with bigger basis sizes. These two are in good agreement for the incident energy $E_{\text {in }}$ up to $2.1 \mathrm{eV}$. One should note that Eq. (14) changes significantly when different basis sets for the molecular structure are used. This is due to the fact that generated positive-energy pseudostates are very different from set to set. Nevertheless, we see that our solution is basis independent when the sufficient number of basis states is taken into consideration.

Having established the robustness of the method, we finally discuss the physical side of our numerical solution. Figure 4 shows the elastic cross sections $\sigma_{v=0}^{N=60}$ together with the excitation cross sections $\sigma_{v=2}^{N=60}, \sigma_{v=4}^{N=60}$, and $\sigma_{v=6}^{N=60}$ [note that only excitation to the states with even $v$ is possible due to

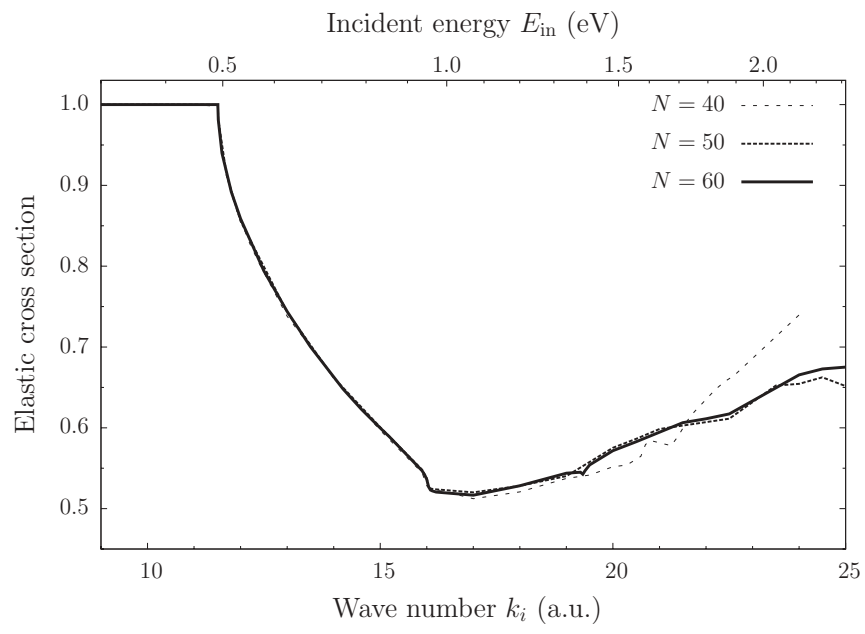

FIG. 3. Cross sections $\sigma_{v=0}^{N}$ of a hydrogen molecule reflecting from a hard-wall potential. Calculations were conducted with three different bases of sizes $N=40,50$, and 60 used to represent the molecular pseudostates. Parameter $\lambda=2$ in all three cases. The basis parameters used for computing the source function $f(\xi)$ were $N_{\text {sf }}=$ 30 and $\lambda_{\mathrm{sf}}=3$.

the factor $\left[\phi_{v}^{*}(\xi)+\phi_{v}^{*}(-\xi)\right]$ in the integrand of the matrix element (8)]. As one can expect no excitation occurs for $E_{\text {in }}<\epsilon_{\nu=2}-\epsilon_{\nu=0} \approx 0.5 \mathrm{eV}$. For larger $E_{i}$ excitation becomes possible and the flux is divided into two channels so that the unitarity condition (18) holds. The elastic cross section $\sigma_{v=0}^{N=60}$ drops down while the excitation cross section $\sigma_{v=2}^{N=60}$ rises up with increasing $E_{\text {in }}$ up to the excitation threshold of the $v=4$ state. At the threshold $\sigma_{v=2}^{N=60}$ has a cusp-like maximum. For larger energies $\sigma_{v=0}^{N=60}$ increases slightly for the whole energy range shown in Fig. 4 while $\sigma_{\nu=2}^{N=60}$ decreases gradually. The behavior of $\sigma_{\nu=4}^{N=60}$ is similar to the behavior of $\sigma_{v=2}^{N=60}$, but with a cusp-like maximum shifted to the next level excitation threshold $E_{\text {in }}=\epsilon_{\nu=6}-\epsilon_{\nu=0} \approx 1.4 \mathrm{eV}$. The other cross sections $\left(\sigma_{v=6}^{N=60}, \sigma_{v=8}^{N=60}\right.$, and $\left.\sigma_{v=8}^{N=60}\right)$ are small in comparison with $\sigma_{\nu=0}^{N=60}$ and $\sigma_{v=2}^{N=60}$. Note that

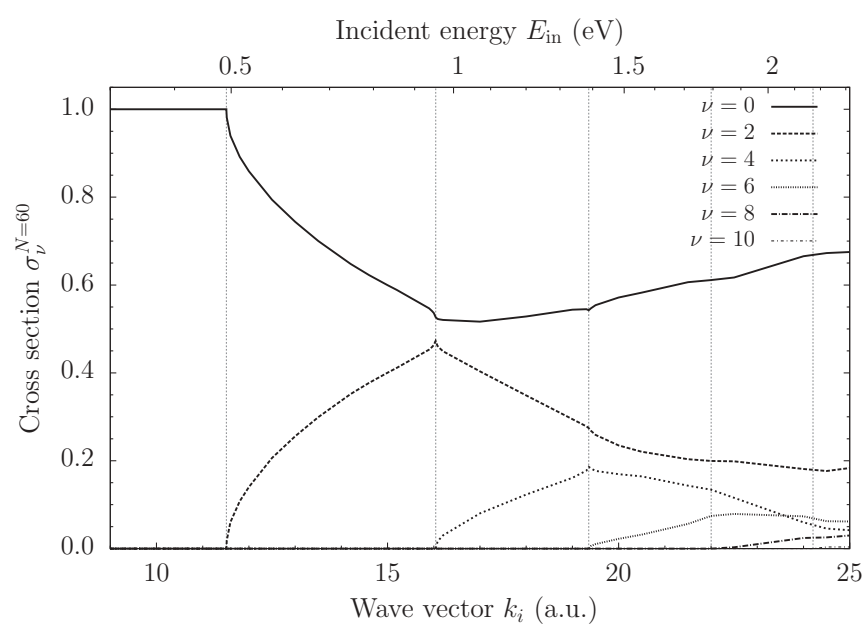

FIG. 4. Elastic cross section $\sigma_{v=0}^{N}$ and excitation cross sections $\sigma_{v}^{N}$ of states with $v=2,4,6$. and 8 versus $E_{i}$. The basis parameters were the same as for Fig. 2. The vertical grid lines show the position of the vibrational excitation thresholds. 
similar cusp-like features at the threshold energies were found for the harmonic interatomic potential by Sato and Kayanuma [13].

One can see from Fig. 4 that the elastic cross section $\sigma_{\nu=0}^{N=60}$ dominates over the other cross sections over the whole range of energy. It rises steadily after reaching the minimum at the threshold energy $E_{\text {in }}=\epsilon_{\nu=4}-\epsilon_{\nu=0} \approx 0.95 \mathrm{eV}$. This is qualitatively different from what was reported by Sato and Kayanuma [13]. They found that the ground state can be completely depopulated due to the collision of a molecule with the hard wall of a finite height for sufficiently large energy $(\approx 0.6 \mathrm{eV})$. The dominant contribution to the total flux was due to the excited channels. One should also mention the results by Kavka et al. [9] for the case of the infinite zero-range binding potential which supports only one weakly bound state and an infinite number of continuum states. Kavka et al. [9] demonstrated that such a molecule "survives" a collision with probability equal to unity when breakup is energetically possible. Thus, we see that our problem of interest demonstrates surprising variability of solutions depending on the choice of the binding potential. This variability is due to the complex interplay of the molecular states coupled by Eq (14). The important point is that a large number of states is required to get a convergent solution even when only a few vibrational channels are open [13].

\section{CONCLUSIONS}

In this paper we studied collinear reflection of a diatomic molecule from a hard-wall potential at the normal angle of incidence. Our method of solution is based on the source-function method developed initially by Masel et al. [14] for the problem of structureless particle scattering from a corrugated hard-wall potential and modified for the description of structured particle excitation due to the collision with the flat hard-wall potential [15]. The second key ingredient of our approach is the continuum discretization technique which allows systematic accounting of both discrete and continuum states of the two-atom system. No approximations for the interatomic interaction potential is required in this method. Our approach can be generalized to treat more complex situations where all six degrees of freedom of a diatomic molecule are taken into account without any simplifications and the hard-wall potential is corrugated.

We have conducted calculations for the case of a molecule bound by the Morse potential. Convergence with increasing basis size is readily demonstrated, establishing the proof of principle of the method. In contrast to what was reported by Sato and Kayanuma [13] for a similar system we found that the elastic cross section has a minimum at $E_{\text {in }}=\epsilon_{\nu=4}-\epsilon_{\nu=0} \approx$ $0.95 \mathrm{eV}$. It poses an interesting question about the behavior of the elastic cross section when the incident energy tends to infinity: To what extent is it possible to depopulate the initial ground state of the molecule? Presently, we do not know the answer to this question. The previous publications on the topic $[9,13]$ deal with two different binding potentials and predict completely different outcomes. The method reported here is very general but it requires numerical solution of the integral equation (14). This becomes problematic for large energies. The Born approximation, which is usually valid for high energies, is not applicable for the long-ranged and divergent hard-wall potential.
[1] M. A. Nielsen and I. L. Chuang, Quantum Computation and Quantum Information (Cambridge University Press, Cambridge, 2000).

[2] L. Förster, M. Karski, J.-M. Choi, A. Steffen, W. Alt, D. Meschede, A. Widera, E. Montano, J. H. Lee, W. Rakreungdet, and P. S. Jessen, Phys. Rev. Lett. 103, 233001 (2009).

[3] O. N. Dorokhov, Zh. Eksp. Teor. Fiz. 98, 646 (1990) [Sov. Phys. JETP 71, 360 (1990)].

[4] D. L. Shepelyansky, Phys. Rev. Lett. 73, 2607 (1994).

[5] Y. Imry, Europhys. Lett. 30, 405 (1995).

[6] N. Saito and Y. Kayanuma, J. Phys.: Condens. Matter 6, 3759 (1994).

[7] C. A. Bertulani, V. V. Flambaum, and V. G. Zelevinsky, J. Phys. G 34, 2289 (2007).

[8] J. Hnybida and M. R. A. Shegelski, Phys. Rev. A 78, 032711 (2008).

[9] J. J. Kavka, D. Kerbrat, and M. R. A. Shegelski, Phys. Rev. A 81, 022708 (2010).
[10] V. L. Bulatov and P. E. Kornilovitch, Europhys. Lett. 71, 352 (2005).

[11] S. Bacca and H. Feldmeier, Phys. Rev. C 73, 054608 (2006).

[12] A. Lemasson, A. Shrivastava, A. Navin, M. Rejmund, N. Keeley, V. Zelevinsky, S. Bhattacharyya, A. Chatterjee, G. de France, B. Jacquot, V. Nanal, R. G. Pillay, R. Raabe, and C. Schmitt, Phys. Rev. Lett. 103, 232701 (2009).

[13] T. Sato and Y. Kayanuma, Europhys. Lett. 60, 331 (2002).

[14] R. I. Masel, R. P. Merrill, and W. H. Miller, Phys. Rev. B 12, 5545 (1975).

[15] A. V. Lugovskoy and I. Bray, Phys. Rev. A 63, 010901(R) (2000).

[16] G. Tepper and D. Miller, Phys. Rev. Lett. 69, 2927 (1992).

[17] P. M. Morse, Phys. Rev. 34, 57 (1929).

[18] I. Bray and A. T. Stelbovics, Phys. Rev. A 46, 6995 (1992).

[19] A. Lugovskoy and I. Bray, Phys. Rev. A 77, 023420 (2008).

[20] C. Schwartz and R. J. Le Roy, J. Mol. Spectrosc. 121, 420 (1987).

[21] A. Bordoni and N. Manini, Int. J. Quantum Chem. 107, 782 (2007). 